\title{
Características y propiedades fisicas de la madera de álamos cultivados en Río Negro, Patagonia Argentina
}

\section{Characteristics and physical properties of poplar woods from Río Negro, Patagonia Argentina}

\author{
Andrea A. Medinal', Agustín G. Baucis', Mariano A. Catalán'2, Ismael R. Andía', Federico Trangoni', Milagros Razquin!, \\ Antonela Pampiglioni' y Anabella Vuillermet ${ }^{1}$
}

Universidad Nacional del Comahue. Asentamiento Universitario San Martín de los Andes. Provincia del Neuquén, Argentina.

\author{
Ministerio de Producción y Turismo de la Provincia \\ del Neuquén. Dirección General de Recursos \\ Forestales. Provincia del Neuquén, Argentina.
}

* Autor de correspondencia. andrepampa@yahoo.com.ar

\section{RESUMEN}

La populicultura en Norpatagonia, principalmente en la provincia de Río Negro, comenzó con el objetivo de establecer cortinas rompevientos para proteger cultivos frutihortícolas en sus valles irrigados. En la actualidad existen $6000 \mathrm{~km}$ de longitud lineal de cortinas y unas 900 ha de plantaciones en macizo de álamo en dicha provincia. El destino de la madera de estos álamos es principalmente la fabricación de embalajes, pero se utiliza también en la construcción, industria del mueble, debobinado y pasta celulósica. No existe información de características y propiedades de esta valiosa materia prima, que es necesaria para optimizar su aprovechamiento y maximizar su valor en diferentes usos. En este trabajo se describen características estéticas, algunas características microscópicas y propiedades físicas de la madera de Populus nigra cv thaisiana, P. alba var bolleana y P. x canadensis 'conti 12', a partir de cinco ejemplares adultos de cada especie, provenientes de cortinas rompevientos de la localidad de Allen, provincia de Rio Negro, Patagonia Argentina. La baja densidad $(<0.40)$ y las características estéticas de la madera de las tres especies determinan su calidad para debobinado, pasta celulósica y fabricación de envases y embalajes. Los valores de contracción volumétrica (11.82\%, 10.32\% y 12.15\%, respectivamente) y del coeficiente de contracción (V: 0.36 - 0.37) indican su ubicación entre las maderas medianamente nerviosas (medianamente estables). Los índices de anisotropía altos (A > 2.3) revelan su baja estabilidad dimensional, indicando la necesidad de realizar correctos secados de la misma para su uso en aberturas y revestimientos.

Palabras Clave: anatomía, anisotropía, contracción, densidad básica, Populus.

\section{ABSTRACT}

Poplar cultivation began in Northern Patagonia, mainly in the Rio Negro province, as windbreaks for the protection of horticultural and fruit crops in its irrigated valleys. At present there are $6000 \mathrm{~km}$ of linear length of forest curtains and about 900 ha of poplar plantations in that province. The wood is used chiefly for the manufacture of containers and packaging, and is also used in building, furniture industry, laminated wood (plywood) and cellulose paste. No information is available on the characteristics and properties of these woods, which would be necessary for optimizing its exploitation and maximizing its value for different uses. This study describes macro and microscopic characteristics and physical properties of the wood of three clones widely used in the area, $P$. nigra cv. thaisiana, $P$. alba var bolleana y $P . \times$ canadensis conti 12 , obtained from five individuals for each clone from windbreaks in Allen, Rio Negro Province, Argentine Patagonia. Low density $(<0.40)$ and macroscopic characteristics of the wood of the three clones determine its quality for lamination, cellulose paste and container and packaging manufacture. They showed medium volumetric contraction $(11.82 \%, 10.32 \% \mathrm{y}$ $12.15 \%$, respectively) and were placed among partially stable to lightly unstable woods (V: $0.36-0.37$ ). High anisotropic indexes (A $>2.3$ ) revealed low dimensional stability, indicating the need for adequate drying processes for its use in windows and linings.

KEYWORDS: anatomy, anisotropy, shrinkage, basic density, Populus. 


\section{INTRODUCCIÓN}

Los álamos en la Argentina representan el tercer cultivo en importancia en el sector forestal después de los pinos y eucaliptos, ocupando unas 50000 ha principalmente distribuidas en las provincias de Buenos Aires, Mendoza y Río Negro (Monteoliva y Senisterra, 2008). En esta última, su cultivo comenzó con el objetivo de establecer cortinas rompevientos para proteger los cultivos frutihortícolas de sus valles irrigados. Tempranamente se implantaron Populus nigra L. cv. italica "álamo criollo" y P. nigra cv. thaisiana "Álamo chileno", siendo este último caracterizado por tener un periodo fenológico extendido, brindando protección prolongada a los cultivos. Posteriormente se fueron implantando otros cultivares de $P$. nigra y del híbrido Euroamericano Populus $x$ canadensis (P. deltoides x P. nigra); de este híbrido el clon 'conti 12' tuvo preferencia por su forma disciplinada y gran rusticidad. Otra especie ampliamente implantada fue Populus alba L. var. bolleana (Lauche) Otto, conocida en la región como "Boleana" (García, 2011; Serventi, 2011).

En la actualidad existen $6000 \mathrm{~km}$ de longitud lineal de cortinas y unas 900 ha de plantaciones en macizo de álamo en esta provincia (Subsecretaría de Desarrollo Foresto Industrial [SSDFI], 2017). La región cuenta con dos plantas de debobinado, una de pasta celulósica y unos 90 aserraderos y fábricas de envases y embalajes (Consejo Federal de Inversiones [CFI], 2012). El destino de la madera de estas cortinas ha sido tradicionalmente la fabricación de envases y embalajes frutihortícolas, pero también se la utiliza en la construcción y la industria del mueble. A modo de ejemplo, en 2011 se consumieron $68565 \mathrm{~m}^{3}$ de madera de álamo en los aserraderos del Alto Valle de Río Negro, alcanzando una producción de $36177 \mathrm{~m}^{3}$, principalmente de cajones, madera aserrada, pallets y bins, tanto con destino local como regional e internacional (CFI, 2012). El empleo de una tecnología inapropiada ha generado la idea de que la madera de álamo es de baja calidad; sin embargo, algunos ejemplos de fabricación de muebles, aberturas y construcción de viviendas prefabricadas con un manejo apropiado de la misma muestran excelentes resultados. El conocimiento de las características y propiedades de estas maderas es esencial para optimizar su aprovechamiento y maximizar su valor en diferentes usos (Williston, 1978; Jozsa y Middleton, 1994). Existe numerosa información sobre la madera de álamos cultivados en Argentina (Diaz, Monteoliva, Alvarez y
Fernández-Tschieder, 2010; Calderón et al., 2011; Cobas y Monteoliva, 2011; Jovanovsky, Robles y Davel, 2011; Cobas, Area y Monteoliva, 2013; Monteoliva, Villegas y Achinelli, 2015) pero escasa o nula de las especies cultivadas en los valles irrigados de la región Norpatagónica. Como lo indican numerosas referencias bibliográficas (Yanchuc, Dancik, y Micko, 1984; Zhang, Yu, Chauret y Koubaa, 2003; Beaudoin, Hernández, Koubaa y Poliquin, 2007; Monteoliva y Senisterra, 2008), la madera de los álamos varía significativamente entre especies y entre sitios, por lo que es necesario conocer las características y las propiedades de la misma para cada especie en cada zona de cultivo.

\section{OBJETIVo}

Descrubir características estéticas, algunas características anatómicas y propiedades físicas de la madera de ejemplares de $P$. nigra cv thaisiana, $P$. alba var boleana y $P . \times$ canadensis 'conti 12 ' cultivados en valles irrigados de Rio Negro, Argentina.

\section{MATERIALES Y MÉTODOS}

Se coleccionaron cinco ejemplares (Tabla 1) de Populus nigra cv thaisiana, P. alba var boleana y P. x canadensis 'conti 12', con buen estado sanitario, de cortinas rompevientos en la localidad de Allen, Provincia de Río Negro (39 0'17.06" S, $67^{\circ}$ 50'41.14"O) (Fig. 1). Se eligieron estas especies por su representatividad en las cortinas de la zona.

El clima de la zona es árido frío, con temperatura media anual $15.5^{\circ} \mathrm{C}$ y precipitación media anual $243.7 \mathrm{~mm}$. Se ubica en la Región Fitogeográfica del Monte (Cabrera, 1976) a 240 m snm, en el complejo aluvial del Río Negro, con suelos xerofluventes típicos, con más de $100 \mathrm{~cm}$ de profundidad, de muy escasa evolución y con un horizonte A bajo en materia orgánica. En profundidad, se superponen distintas capas de acumulación fluvial de antiguos planos aluviales (Bran, 2014).

De cada ejemplar se extrajo una troza basal de $30 \mathrm{~cm}$ desde la cual se obtuvo madera para la elaboración de muestras para descripción de características estéticas, de muestras microscópicas para estudios anatómicos y de probetas para ensayos de propiedades físicas (densidad, cambios dimensionales y humedad del árbol vivo). De cada ejemplar se coleccionó material de herbario de referencia depositado en el Herbario del 
Asentamiento Universitario San Martín de los Andes (Ausma) de la Universidad Nacional del Comahue (Disp.CD-AUSMA $21 / 14)$.

Las muestras macroscópicas de madera se elaboraron de 18 $\mathrm{cm} \times 10 \mathrm{~cm} \times 2 \mathrm{~cm} \mathrm{y}$, a partir ellas, se describieron las siguientes características estéticas: color (Munsell Color Company, 1990), aroma, brillo, textura, grano y veteado (Tortorelli, 2009). Las mismas fueron depositadas en la Xiloteca "Luis F. Lerín" del Asentamiento Universitario San Martín de los Andes de la Universidad Nacional del Comahue (Medina y Andía, 2016).

Para los estudios anatómicos se realizaron tres preparados microscópicos y macerados por ejemplar, mediante la técnica de Franklin (1945). Se midieron las siguientes variables: longitud de fibras, longitud de elementos de vaso, diámetro de poros, número de poros por milímetro cuadrado, diámetro externo de fibras, diámetro interno de fibras, espesor de pared de las fibras y porcentaje de tejidos, siguiendo las recomendaciones de Muñiz y Coradin (1991), midiendo por lo menos 25 elementos por variable. El cálculo de porcentaje de tejidos se realizó con la metodología propuesta por Quirk y Smith (1975). Se utilizó microscopio binocular con ocular micrométrico. Para las clasificaciones de las variables se siguió a Tortorelli (2009).

Para la determinación de la densidad de la madera se elaboraron 10 especímenes de $2 \mathrm{~cm} \times 2 \mathrm{~cm} \times 2 \mathrm{~cm}$, por cada árbol. De cada espécimen se registró el volumen en estado verde $(\mathrm{Vv})$; posteriormente se llevaron a peso constante en estufa (103 ${ }^{\circ} \mathrm{C} \pm 2{ }^{\circ} \mathrm{C}$ ) y se determinaron el peso (Po) y el volumen anhidro (Vo), para obtener la densidad relativa básica (Db) y la densidad aparente anhidra (Do) con las siguientes fórmulas:

Densidad relativa básica $(\mathrm{Db})=(\mathrm{Po} / \mathrm{Vv})$

Densidad aparente anhidra (Do) $=$ Po $/$ Vo

El volumen se determinó por desplazamiento de agua sobre balanza electrónica según lo recomendado por Williamson y Wiemann (2010).

Para los ensayos de cambios dimensionales se elaboraron seis especímenes libres de defectos por cada árbol (dos tangenciales, dos radiales y dos axiales) sus dimensiones fueron $10 \mathrm{~cm} \times 2 \mathrm{~cm} \times 2 \mathrm{~cm}$. De cada espécimen en condición verde se
TABLA 1. Diámetro a la altura del pecho (DAP) y altura total de los ejemplares coleccionados.

\begin{tabular}{|c|c|c|c|}
\hline Ejemplar & Especie & $D A P(\mathrm{~cm})$ & $\begin{array}{l}\text { Altura } \\
\text { total (m) }\end{array}$ \\
\hline 1 & P. alba var bolleana & 36.4 & 17.8 \\
\hline 2 & P. alba var bolleana & 37 & 20 \\
\hline 3 & P. alba var bolleana & 37.6 & 22 \\
\hline 4 & P. alba var bolleana & 37.8 & 23.1 \\
\hline 5 & P. alba var bolleana & 40.2 & 23.5 \\
\hline 6 & Populus nigra cv thaisiana & 42 & 27.2 \\
\hline 7 & Populus nigra cv thaisiana & 44.7 & 21.2 \\
\hline 8 & Populus nigra cv thaisiana & 45 & 22 \\
\hline 9 & Populus nigra cv thaisiana & 45.5 & 25 \\
\hline 10 & Populus nigra cv thaisiana & 42 & 28 \\
\hline 11 & P. x canadensis conti 12 & 44.5 & 28.1 \\
\hline 12 & P. x canadensis conti 13 & 44 & 25 \\
\hline 13 & P. $x$ canadensis conti 14 & 42 & 29 \\
\hline 14 & P. $x$ canadensis conti 15 & 44 & 29 \\
\hline 15 & P. $x$ canadensis conti 16 & 42.2 & 26.5 \\
\hline
\end{tabular}

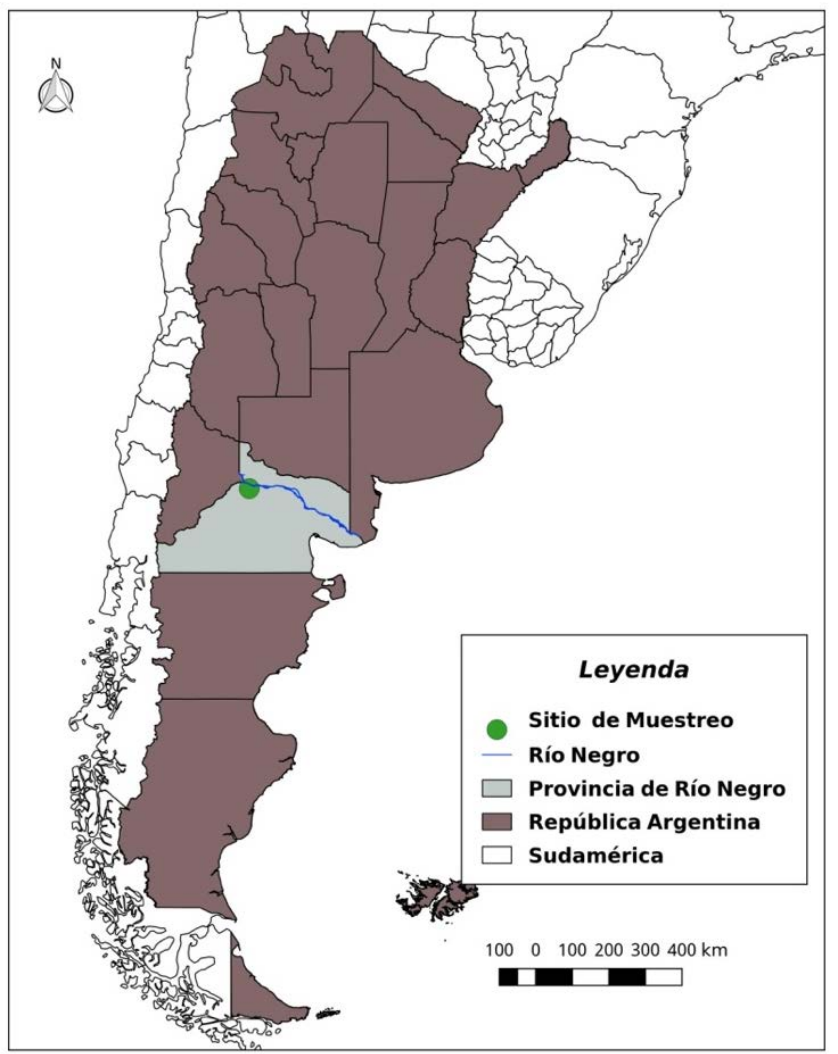

FIGURA 1. Ubicación del sitio de colección de los árboles estudiados. 
obtuvieron: peso $(\mathrm{Pv})$, volumen $(\mathrm{Vv})$, longitud tangencial $(\mathrm{Tv})$, longitud radial $(\mathrm{Rv})$ y longitud axial $(\mathrm{Av})$, para determinar las contracciones: volumétrica total $(\mathrm{CVt})$, tangencial $(\mathrm{CT})$, radial (CR) y axial (CA). Posteriormente, los especímenes se llevaron a peso constante (estado anhidro) en estufa $\left(103{ }^{\circ} \mathrm{C} \pm 2{ }^{\circ} \mathrm{C}\right)$ y se registró peso (Po), volumen (Vo) y longitudes tangencial (To), radial (Ro) y axial (Ao). Por último, se llevaron a estado seco al aire y se obtuvo peso $(\mathrm{Pa})$ y volumen $(\mathrm{Va})$, con el fin de estimar contenido de humedad $(\mathrm{CHa})$ y contracción parcial volumétrica (CVp) para el cálculo del coeficiente de contracción volumétrica $(\mathrm{Vv})$. Este coeficiente expresa cuánto se contrae una madera al perder $1 \%$ de su humedad. El punto de saturación de la fibra (PSF) se calculó según lo propuesto por Fuentes-Salinas (2000). El índice de anisotropía (A), indicador del grado de estabilidad dimensional de la madera, se calculó como el cociente entre CT y CR.

Para los cálculos se utilizaron las siguientes fórmulas:

$$
\begin{aligned}
& \mathrm{CVt}(\%)=(\mathrm{Vv}-\mathrm{Vo} / \mathrm{Vv}) 100 \\
& \mathrm{CT}(\%)=(\mathrm{Tv}-\mathrm{To} / \mathrm{Tv}) 100 \\
& \mathrm{CR}(\%)=(\mathrm{Rv}-\mathrm{Ro} / \mathrm{Rv}) 100 \\
& \mathrm{CA}(\%)=(\mathrm{Av}-\mathrm{Ao} / \mathrm{Av}) 100 \\
& \mathrm{CHa}(\%)=(\mathrm{Pa}-\mathrm{Po} / \mathrm{Po}) 100 \\
& \mathrm{CVp}(\%)=(\mathrm{Vh}-\mathrm{Vo} / \mathrm{Vh}) 100 \\
& \mathrm{Vv}=\mathrm{CVp} / \mathrm{CHa} \\
& \mathrm{PSF}(\%)=\mathrm{CVt} / \mathrm{Vv}
\end{aligned}
$$

El contenido de humedad del árbol vivo (CHv) se estimó utilizando la siguiente fórmula:

$\mathrm{CHv}(\%)=(\mathrm{Pv}-\mathrm{Po} / \mathrm{Po}) 100$

\section{Resultados}

\section{Características estéticas}

La madera de las especies analizadas presentó colores claros, con escasa o nula diferencia entre albura y duramen, ausencia de aroma y brillo, textura fina y homogénea, grano derecho y veteado liso (Tabla 2, Fig. 2).

\section{Características microscópicas analizadas}

Las tres especies presentaron madera con anillos de crecimiento demarcados por la compresión tangencial de las fibras del leño tardío, porosidad difusa, vasos solitarios y múltiples radiales, muy numerosos, medianos en longitud y de pequeños en diámetro y fibras libriformes de longitud media, finas de diámetro y de paredes delgadas. El parénquima axial observado fue del tipo paratraqueal difuso muy escaso (menos de $0.2 \%$ del tejido leñoso), con células aisladas ubicadas en el inicio del anillo de crecimiento. Los radios leñosos fueron numerosos, uniseriados y bajos, constituyendo hasta 15\% del tejido (Tabla 3).

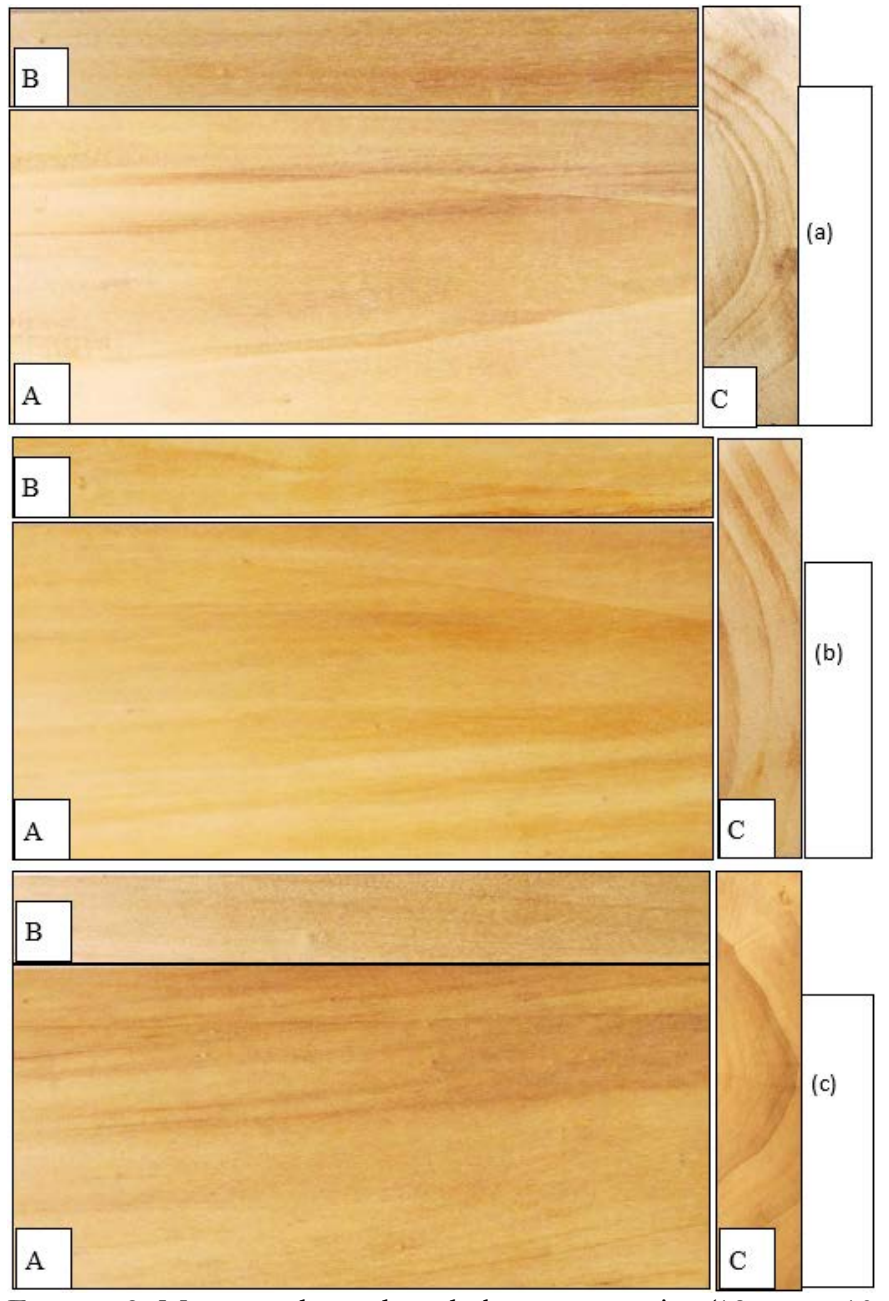

FIGURA 2. Muestras de madera de las tres especies $(18 \mathrm{~cm} \times 10$ $\mathrm{cm} \times 2 \mathrm{~cm}$ );

a) P. x canadensis conti 12; (b) P. alba var bolleana; (c) P. nigra cv thaisiana A: sección tangencial; B: sección radial y C: sección transversal. 
TABLA 2. Características estéticas de la madera de las tres especies $(n=5)$.

\begin{tabular}{llll}
\hline & Especie & & \\
& P.nigra cv thaisiana & P. alba var bolleana & P.x canadensis conti 12 \\
\hline Color albura & Marrón muy pálido* & Amarillo pálido** & Amarillo pálido \\
Color duramen & Marrón muy pálido & Marrón muy pálido & Marrón muy pálido \\
Aroma & Ausente & Ausente & Ausente \\
Brillo & Ausente & Ausente & Ausente \\
Grano & Derecho & Derecho & Derecho \\
Textura & Fina y homogénea & Fina y Homogénea & Fina y homogénea \\
Veteado & Liso & Liso & Liso \\
\hline
\end{tabular}

*HUE 1 O Y R 8/2; **HUE 2.5 Y $8 / 2$

TABLA 3. Valores medios, desviación estándar y coeficiente de variación de características anatómicas analizadas $(\mathrm{n}=375)$.

\begin{tabular}{|c|c|c|c|c|c|c|c|c|c|}
\hline & \multicolumn{9}{|l|}{ Especie } \\
\hline & \multicolumn{3}{|c|}{ P.nigra cv thaisiana } & \multicolumn{3}{|c|}{ P. alba var bolleana } & \multicolumn{3}{|c|}{ P. x canadensis conti 12} \\
\hline & Media & $\mathrm{DE}$ & $C V$ & Media & DE & $C V$ & Media & DE & $C V$ \\
\hline Long. Fibras ( $\mu \mathrm{m})$ & 1028.0 & 144.3 & 14.0 & 1137.0 & 158.9 & 14.0 & 1114.0 & 125.7 & 11.3 \\
\hline Long. Vasos ( $\mu \mathrm{m})$ & 508.4 & 44.1 & 8.7 & 587.7 & 75.5 & 12.8 & 553.8 & 92.4 & 16.7 \\
\hline Diám. Poros ( $\mu \mathrm{m})$ & 61.5 & 11.0 & 17.8 & 58.3 & 8.9 & 15.2 & 70.4 & 9.9 & 14.1 \\
\hline $\mathrm{N}^{\circ}$ poros $\mathrm{mm}^{-2}$ & 31.7 & 11.0 & 34.6 & 31.5 & 12.4 & 39.3 & 26.4 & 5.1 & 19.5 \\
\hline Diám. Fibras $(\mu \mathrm{m})$ & 19.7 & 1.8 & 9.3 & 21.6 & 2.5 & 11.7 & 23.3 & 3.4 & 14.6 \\
\hline Esp. pared Fibras $(\mu \mathrm{m})$ & 3.9 & 0.7 & 17.5 & 4.2 & 0.7 & 16.2 & 4.6 & 0.7 & 15.3 \\
\hline Poros (\%) & $30 ., 3$ & 7.0 & 23.0 & 27.8 & 6.9 & 24.9 & 32.0 & 5.7 & 17.7 \\
\hline Fibras (\%) & 54.5 & 8.5 & 15.6 & 58.3 & 7.8 & 13.5 & 57.2 & 10.5 & 18.3 \\
\hline Radios leñosos (\%) & 15.1 & 4.3 & 28.3 & 13.7 & 4.5 & 28.0 & 12.6 & 4.4 & 29.0 \\
\hline
\end{tabular}

\section{Propiedades físicas}

Las tres especies presentaron madera con densidad baja (Tabla 4), contracción volumétrica mediana, índices de anisotropía altos (A) y coeficiente de contracción volumétrica (V) elevados (Fig. 3, Tabla 5).

\section{Discusión y conclusiones}

Las características estéticas de la madera de las tres especies (Fig. 2, Tabla 2) definen, entre otras, su calidad para obtención de chapas por debobinado y para la fabricación de cajones y embalajes para alimentos (Tortorelli, 2009). La presencia de anillos de crecimiento levemente demarcados determina el veteado liso en las caras tangenciales y radiales. Los poros en disposición difusa, numerosos y de diámetro pequeño, resultan en su textura fina y homogénea.
El porcentaje de tejidos, la longitud de fibras y la longitud de elementos de vaso de la madera de los álamos estudiados concuerdan con los valores registrados para álamo por Panshin y de Zeeuw (1980). Dichos autores afirman que la densidad relativa de estas maderas está fuertemente influenciada por la relación poros/fibras, así como por el diámetro y el espesor de las paredes de dichas células. Las tres especies estudiadas presentan madera de baja densidad (densidad relativa básica $\leq 0.40$ ) (International Association of Wood Anatomists [IAWA], 1989) (Tabla 3). La densidad de la madera es considerada como una de las propiedades físicas más importantes debido a la relación que tiene con otras propiedades y con sus posibles usos. Es por eso que es generalmente utilizada como indicador de propiedades mecánicas y otras características físicas y anatómicas de la madera (AguilarRodríguez, Abundiz-Bonilla y Barajas-Morales, 2001). 
TABLA 4. Valores medios, desviación estándar y coeficiente de variación de la densidad aparente anhidra y la densidades relativa básica de la madera de las tres especies $(\mathrm{n}=50)$.

\begin{tabular}{|c|c|c|c|c|c|c|c|c|c|}
\hline & \multicolumn{8}{|c|}{ Especie } & \\
\hline & \multicolumn{3}{|c|}{ P.nigra cv thaisiana } & \multicolumn{3}{|c|}{ P. alba var bolleana } & \multicolumn{3}{|c|}{ P. $x$ canadensis conti 12} \\
\hline & Media & $\mathrm{DE}$ & $\mathrm{CV}$ & Media & $\mathrm{DE}$ & CV & Media & $\mathrm{DE}$ & CV \\
\hline Densidad relativa básica & 0.32 & 0.02 & 6.25 & 0.36 & 0.04 & 11.11 & 0.35 & 0.02 & 5.71 \\
\hline Densidad anhidra $\left(\mathrm{g} \mathrm{cm}^{-3}\right)$ & 0.37 & 0.02 & 5.41 & 0.42 & 0.05 & 11.90 & 0.42 & 0.03 & 7.14 \\
\hline
\end{tabular}

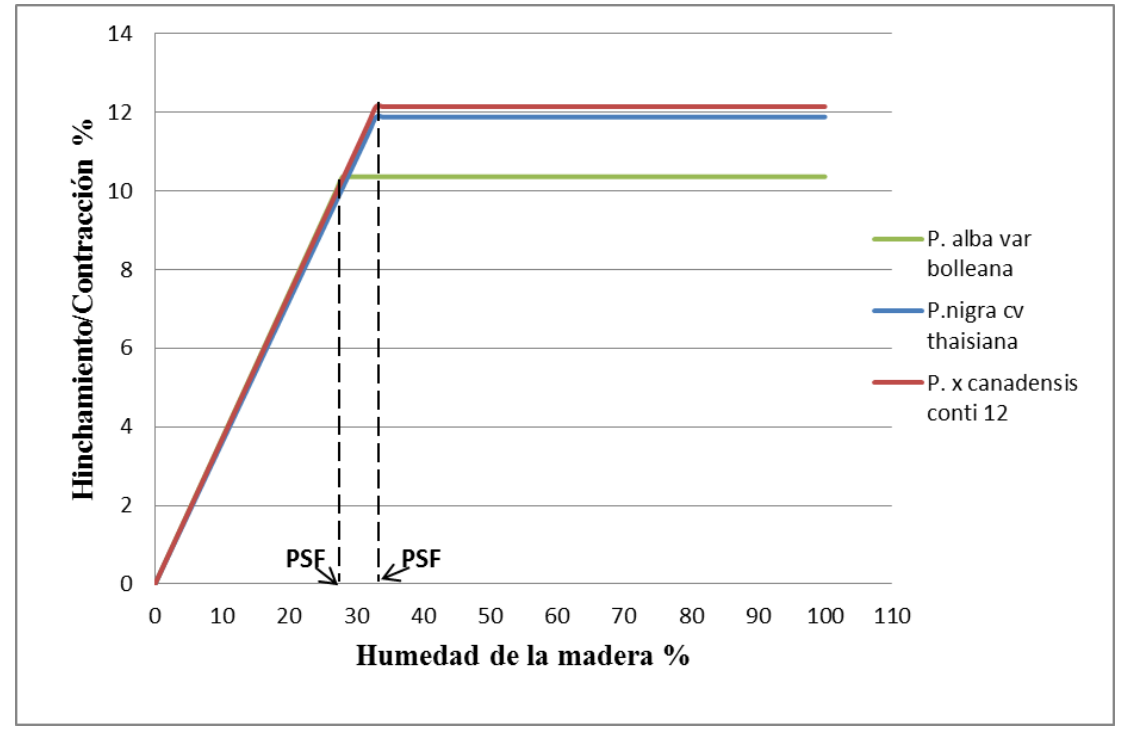

FIGURA 3. Curva de hinchamiento/contracción de la madera de las tres especies analizadas en relación con su contenido de humedad.

TABLA 5. Valores de cambios dimensionales de la madera y humedad del árbol vivo $(\mathrm{n}=30)$.

\begin{tabular}{lccc}
\hline & \multicolumn{4}{c}{ Especie } \\
\hline & $\begin{array}{c}\text { P.nigra cv } \\
\text { thaisiana }\end{array}$ & $\begin{array}{c}\text { P. alba var } \\
\text { bolleana }\end{array}$ & $\begin{array}{c}\text { P.x canadensis } \\
\text { conti } 12\end{array}$ \\
\hline Contracción máxima Tangencial (\%) & 8.34 & 7.41 & 8.48 \\
\hline Contracción máxima Radial (\%) & 3.24 & 2.75 & 3.67 \\
\hline Contracción máxima Volumétrica (\%) & 11.82 & 10.32 & 12.15 \\
\hline Coef. Contracción Volumétrica (V) & 0.36 & 0.37 & 0.37 \\
\hline Índice Anisotropía (A) & 2.57 & 2.69 & 2,31 \\
\hline PSF (\%) & 33 & 28 & 33 \\
\hline H. del árbol vivo (\%) & 211.2 & 122.7 & 179 \\
\hline
\end{tabular}


Las maderas de las tres especies presentan contracción volumétrica mediana (Tabla 4), ubicándose, por el valor que arrojó el coeficiente de contracción volumétrica (V), entre las maderas medianamente nerviosas $(\mathrm{V}=0.55$ - 0.35) (Tuset et al., 2008). Los índices de anisotropía (A) altos (Tabla 4) revelan baja estabilidad dimensional de estas maderas (Andía y Keil, 2004) indicando la necesidad de realizar cuidadosos y correctos secados de la misma (Tuset et al., 2008). Según tipificación propuesta por Coronel (1994), las maderas de los álamos estudiados se ubican entre las de estabilidad dimensional regular a mala. Los valores de contracción volumétrica e índice de anisotropía concuerdan con los hallados para maderas de álamos por Balatinecz y Kretschmann (2001). La estabilidad dimensional de la madera "en servicio", llamada comúnmente "juego de la madera”, caracteriza a las maderas por su comportamiento ante cambios de humedad y tiene especial importancia en construcciones de marcos de puertas y ventanas, hojas de ventanas y revestimientos en general. Existen trabajos sobre densidad y contracción de la madera de álamos cultivados en Patagonia, Argentina, provenientes de ejemplares de cortinas rompevientos de $P$ nigra $c v$ italica, $P$. nigra $c v$ Sehuil y P. trichocarpa de la provincia de Chubut en Argentina, con valores de densidad anhidra de $0.31 \mathrm{~g} \mathrm{~cm}^{-3}, 0.31 \mathrm{~g} \mathrm{~cm}^{-3}$ y $0.32 \mathrm{~g}$ $\mathrm{cm}^{-3}$, respectivamente, y valores de contracción volumétrica mediana e índices de anisotropía altos (Jovanovsky et al., 2011), similares a los encontrados en este trabajo. También Otaño, Peri, Luna, Keil y Díaz (2002) encontraron densidades anhidras de la madera de $P$ nigra cv itálica de la provincia de Santa Cruz de 0.33 $\mathrm{g} / \mathrm{cm}^{-3}$ a $0.36 \mathrm{~g} / \mathrm{cm}^{-3}$ dependiendo de la localidad.

El crecimiento en diámetro de los fustes de árboles plantados en cortinas suele desarrollarse manifestando una sección transversal de forma elíptica a causa de la ubicación localizada del follaje en el espacio disponible durante su crecimiento. Esta circunstancia, sumada a factores externos como la dirección predominante del viento, puede influir en las características de la madera, dando lugar a la formación de leño de tensión. Jovanovsky et al. (2011) analizaron la variación de la densidad y la contracción de la madera entre leño normal y de tensión álamo, no encontrando diferencias significativas.

El Punto de saturación de las fibras (PSF) de la madera en general es un valor teórico de $30 \%$, y que en la práctica puede variar de una especie a otra entre 25\% y 41\% de humedad. En dicho punto no hay presencia de agua libre, pero las paredes celulares se encuentran completamente saturadas de agua. Este punto tiene gran importancia práctica, ya que por encima de él permanecen constantes las dimensiones de la pieza y las propiedades mecánicas de las maderas (Andía y Keil, 2004). Por debajo de él, se produce una merma en las dimensiones y una mejora de la mayoría de las propiedades de resistencia mecánica. También tiene importancia notable en el consumo energético por unidad de volumen de agua a evaporar, ya que el agua ligada es retenida con mayor energía que el agua libre (Tuset et al., 2008). El alto contenido de humedad de la madera del árbol vivo registrado en el presente trabajo es característico de las especies de Populus; esta particularidad permite que en la práctica del debobinado, e incluso en la del guillotinado (rebanado), no sea necesario el calentamiento de la madera por sistemas de vapor o agua caliente (Balatinecz y Kretschmann, 2001). Esta característica también influye en los costos de transporte cuando este se realiza por peso (t) de la madera.

Resultados de estudios en desarrollo de variabilidad y de propiedades mecánicas de estas maderas aportaran avance significativo en su conocimiento. En forma posterior será necesario ensayar programas de secado para éstas maderas en pos de un aprovechamiento óptimo. Si bien hasta el momento el álamo no ha sido usado masivamente en Argentina como madera estructural, se infiere que los valores anatómicos y físicos que se presentan en este trabajo no la descartan a priori para ese destino.

\section{RECONOCIMIENTOS}

Se agradece el apoyo de técnico forestal Nicolás Mantilaro en la elaboración de las muestras macroscópicas y probetas para ensayos de propiedades físicas. La colaboración del técnico regional de la Subsecretaria de Desarrollo Foresto-Industrial del Ministerio de Agroindustria de la Nación Julio García, al consultor privado e ingeniero agrónomo Norberto Serventi y al presidente de la Cámara de Forestadores y Empresarios Madereros y Afines de Norpatagonia Nazareno Olivetti, en la elección de las cortinas de álamo y los contactos para la colección del material y su procesado inicial. A la técnica forestal. (MSc) María Cristina Frugoni por descripción de suelos. A la Dirección General de Recursos Forestales de la Provincia del Neuquén en 
la figura de su director, Uriel Mele, por el apoyo logístico. Al MSc. Sergio I. Tiranti por traducción y revisión.

\section{REFERENCIAS}

Aguilar-Rodríguez, S., Abundiz-Bonilla, L., \& Barajas-Morales, J. (2001). Comparación de la gravedad específica y características anatómicas de la madera de dos comunidades vegetales de México. Anales del Instituto de Biología. Serie Botánica, 72(2), 171-185.

Andía, I., \& Keil, G. (2004). Propiedades físicas de la madera. Publicación docente $\mathrm{n}^{\circ}$ 01/04. Cátedra de Tecnología de la Madera. Universidad Nacional del Comahue.

Balatinecz, J. J., \& Kretschmann, D. E. (2001). Properties and utilization of poplar wood. Poplar culture in North America. Ottawa, Canada: NRC Research Press.

Beaudoin, M., Hernández, R. E., Koubaa, A., \& Poliquin, J. (2007). Interclonal, intraclonal and within-tree variation in wood density of poplar hybrid clones. Wood and Fiber Science, 24(2), 147-153.

Bran, D. E. (2014). Inventario integrado de los recursos naturales de la provincia de Río Negro. Provincia de Río Negro, Argentina: Ediciones INTA.

Cabrera, A. L. (1976). Regiones fitogeográficas de la Argentina. Enciclopedia Argentina de Agricultura y Jardinería. Tomo II. Buenos Aires, Argentina.

Calderón, A. D., Roig, F. A., Zanetti, R. P., Aguado, E. G., Furlani, A. M., \& Martínez, F. G. (2011). Características físico-mecánicas de maderas de clones de álamo ensayadas en la provincia de Mendoza, República Argentina. $3^{\text {er }}$ Congreso Internacional de Salicaceas en Argentina. Neuquén, Argentina.

Cobas, A. C., \& Monteoliva, S. (2011). Patrones de variación axialy radial de densidad y longitud de fibras en Populus asociados a la formación de madera juvenil y madura. $3^{\text {er }}$ Congreso Internacional de Salicaceas en Argentina. Neuquén, Argentina.

Cobas, A. C., Area, M. C., \& Monteoliva, S. (2013). Transición de madera juvenil a madura en Populus deltoides implantado en Buenos Aires, Argentina. Madera Ciencia y Tecnología, 15, 223-234. doi: 10.4067/S0718-221X2013005000018

Consejo Federal de Inversiones [CFI]. (2012). Censo de Industrias de la Madera de la Provincia del Neuquén y zonas de influencia del Alto V alle del Río Negro. Neuquén: CFI.

Coronel, E.O. (1994). Fundamentos de las propiedades físicas y mecánicas de la madera. Aspectos teóricos y prácticos de las propiedades y sus aplicaciones. Santiago del Estero, Argentina: Editorial El Liberal.

Diaz, G., Monteoliva, S., Alvarez, J., \& Fernández-Tschieder, E. (2010). Populus deltoides'Australiano 129/60': variación axial de la densidad y desarrollo de un modelo predictivo de la densidad del árbol completo. Bosque, 31(1): 65-72. doi: 10.4067/S071792002010000100008
Franklin, G. L. (1945). Preparation of thin sections of synthetic resins and wood-resin composites, and a new macerating method for wood. Nature, 155(3924), 51.doi: 10.1038/155051a0

Fuentes-Salinas, M. (2000). Estimación del punto de saturación de la fibra (PSF) de las maderas. Revista Chapingo Serie Ciencias Forestales y del Ambiente, 6(1), 79-81.

García, J. 2011. El cultivo de las Salicáceas en los valles irrigados de la Patagonia, pasado, presente y futuro. $3^{\text {er }}$ Congreso Internacional de Salicaceas en Argentina. Neuquén, Argentina.

International Association of Wood Anatomists [IAWA]. (1989). IAWA List of Microscopic features for hardwood identification. IAWA Bulletin, 10(3), 219-332. doi: 10.1163/22941932-90000496

Jovanovsky, A., Robles, G., \& Davel, M. (2011). Propiedades físicas de la madera de Populus sp proveniente de cortinas forestales plantadas en Esquel, Chubut, Argentina. $3^{\text {er }}$ Congreso Internacional de Salicaceas en Argentina. Neuquén, Argentina.

Jozsa, L. A., \& Middleton, G. R. (1994). A discussion of wood quality attributes and their practical implications. Vancouver B.C.: Forintek Canada Corp. Western Laboratory.

Medina, A. A., \& Andía, I. R. (2016). Valiosa colección de maderas de valor didáctico y científico en la Universidad Nacional del Comahue. Desde la Patagonia Difundiendo saberes, 13(21), 22-26.

Monteoliva, S., \& Senisterra, G. (2008). Efecto del sitio, el origen y el clon sobre el crecimiento y propiedades de la madera de Populus. Investigación Agraria: Sistemas y Recursos Forestales, 17(3), 261-270.

Monteoliva, S., Villegas, M. S., \& Achinelli, F. G. (2015). Short-term and long-term effects of weed control and fertilization on growth and wood anatomy of a Populus deltoides clone. Forest Systems, 24(1), 15. doi: $10.5424 /$ fs / 2015241-05077

Munsell Color Company (1990). Munsell soil color charts. Baltimore, Maryland: Munsell Color Co.

Muñiz, G., \& Coradin, V. (1991). Norma de procedimientos en estudios de anatomía da madeira. II Gimnospermae Comissae de estudios. CE: 11.01.07.002. Associação Brasileira de Normas Técnicas. Serie Técnica. Brasilia. Laboratorio de Produtos Florestais.

Otaño, M. E., Peri, P. L., Luna, M. L., Keil, G. D., \& Díaz, B. G. (2002). Comportamiento de la madera de Populus nigra cv italica ante la preservación con creosota y CCA. Investigación Agraria. Sistemas y Recursos Forestales, 11(2), 325-338.

Panshin, A. J., \& de Zeeuw, C. (1980). Textbook of wood technology. Nueva York, Estados Unidos: McGraw-Hill.

Quirk, J. T., \& Smith, D. (1975). Comparison of dual linear and dot-grid eyepiece methods for estimating wood properties of Douglas-fir. Madison, Wisconsin: Wood Sciences and Forest Products Resources Society. 
Serventi, N. (2011). Las cortinas forestales en los valles irrigados de Norpatagonia. $3^{\text {er }}$ Congreso Internacional de Salicaceas en Argentina. Neuquén, Argentina.

Subsecretaría de Desarrollo Foresto Industrial [SSDFI]. (2017). Inventario nacional de plantaciones forestales bajo riego. Región Patagónica: Ministerio de Agroindustrias, Presidencia de la Nación.

Tortorelli, L. A. (2009). Maderas y bosques argentinos (2a edición). Buenos Aires: Orientación Gráfica Editora.

Tuset, R., Durán, F., Mantero, C., Baillod, G., Aber, A., Bothig, S., \& Ono, A. (2008). Manual de maderas comerciales, equipos y procesos de utilización (2a edición). Volumen II. Argentina: Editorial Agropecuaria Hemisferio Sur.

Williamson G. B., \& Wiemann, M. C. (2010). Measuring wood specific gravity correctly. American Journal of Botany, 97(3), 519-524. doi:10.3732/ajb.0900243

Williston, M. (1987). Lumber manufacturing: the design and operation of sawmills and planer mills. Estados Unidos: Miller Freeman Publications Inc.

Yanchuc, A. D., Dancik, B. P., \& Micko, M. M. (1984). Variation and heritability of wood density and fibre length of trembling aspen in Alberta, Canada. Canadá. Silvae Genetica, 33(1), 11-16.
Zhang, S. Y., Yu, Q., Chauret, G., \& Koubaa, A. (2003). Selection for Both Growth and Wood Properties in Hybrid Poplar Clones. Forest Science, 49(6), 901-908.

Manuscrito recibido el: 24 de octubre de 2016

Aceptado el: 12 de septiembre de 2017

Publicado el: 8 de marzo de 2018

Este documento se debe citar como:

Medina, A. A, Baucis, A. G., Catalán, M. A., Andía, I. R., Trangoni, F., Razquin, M., Pampiglioni, A., \& Vuillermet, A. (2018). Características y propiedades físicas de la madera de álamos cultivados en Río Negro, Patagonia Argentina. Madera y Bosques, 24(1), e2411434. doi: $10.21829 /$ myb.2018.2411434

Madera y Bosques por Instituto de Ecología, A.C. se distribuye bajo una Licencia Creative Commons Atribución-NoComercialCompartirlgual 4.0 Internacional. 